\title{
Streptococcus pyogenes upper respiratory infection and atopic conditions other than asthma: a retrospective cohort study
}

\author{
*Young J Juhna, Diana Frey', Xujian Lic, Robert Jacobson ${ }^{\mathrm{a}}$ \\ a Department of Community Pediatric \& Adolescent Medicine, Mayo Clinic, Rochester, Minnesota, USA \\ ${ }^{\mathrm{b}}$ Mayo Medical School, Mayo Clinic, Rochester, Minnesota, USA \\ c Department of Biomedical Statistics \& Informatics, Mayo Clinic, Rochester, Minnesota, USA
}

Originally received 9th February 2011; resubmitted 20th May 2011; revised 23rd August 2011; accepted 13th September 2011; online 23rd January 2012

\begin{abstract}
Background: Patients with asthma have an increased risk of Streptococcus pyogenes infection compared with those without asthma. It is unknown whether this is true for children with other atopic conditions such as atopic dermatitis or allergic rhinitis.

Aims: To determine the risk of developing $S$. pyogenes infections of the upper respiratory tract in children and adolescents with atopic dermatitis and/or allergic rhinitis.

Methods: We conducted a retrospective cohort study that followed a convenience sample of 340 healthy children. Atopic dermatitis or eczema and allergic rhinitis or hay fever were determined based on a physician diagnosis documented in medical records. All laboratory test results of cultures, rapid antigen detection, and polymerase chain reaction tests for $S$. pyogenes infections during the first 18 years of life were collected to compare the incidence of $S$. pyogenes infections between children with and without a physician diagnosis of atopic conditions. A Poisson regression was fit to determine the association between asthma and S. pyogenes infections, controlling for other covariates including asthma.

Results: Of the 340 subjects, 327 were eligible for the study. Of these 327 subjects, 143 (44\%) had atopic conditions other than asthma. The incidence of $S$. pyogenes infections in children with atopic conditions other than asthma and those without atopic conditions was 0.24 per person-year and 0.18 per person-year, respectively. The adjusted risk ratios for allergic rhinitis and atopic dermatitis were 1.36 (95\% $\mathrm{Cl} 1.07$ to $1.66, \mathrm{p}=0.011)$ and $1.30(95 \% \mathrm{Cl} 0.98$ to $1.71, \mathrm{p}=0.06)$, respectively, controlling for asthma and other covariates.

Conclusions: In addition to asthma, allergic rhinitis but not atopic dermatitis is associated with an increased risk of $S$. pyogenes upper respiratory tract infections.

(C) 2012 Primary Care Respiratory Society UK. All rights reserved.

YJ Juhn et al. Prim Care Respir J 2012; 21(2): 153-158

http://dx.doi.org/10.4104/pcrj.2011.00110
\end{abstract}

Keywords atopic dermatitis, allergic rhinitis, children, Streptococcus pyogenes, infection

See linked editorial by Yusuf on pg 126

\section{Introduction}

Streptococcus pyogenes infections are a major cause of morbidity and mortality worldwide with an estimated 500,000 deaths, most of which are attributable to invasive infection, acute rheumatic fever, and subsequent rheumatic heart disease. ${ }^{1}$ They cause a spectrum of diseases from sore throat to fatal necrotising fasciitis. Approximately 7.3 million outpatient physician visits attributable to sore throat occur each year among children in the USA, and S. pyogenes is responsible for $15-36 \%$ of cases. ${ }^{2}$ Data from the 2004 World Health Organization report show that death from $S$. pyogenes infection was ranked ninth among the top 10 causes of death in the world. ${ }^{3} \mathrm{~A}$ recent report suggests that $\mathrm{S}$. pyogenes infection was the third most common organism causing pulmonary bacterial co-infection in patients with fatal cases of 2009 novel H1N1 influenza. ${ }^{4}$ Each year, 600 million new cases of $\mathrm{S}$. pyogenes pharyngitis, 500,000 deaths worldwide, ${ }^{5}$ and an estimated 8,950-11,500 cases of invasive S. pyogenes infections occur in the USA resulting in 1,050-1,850 deaths. ${ }^{6}$ Much of this burden of $\mathrm{S}$. pyogenes infection is attributable to symptomatic infection and asymptomatic

\footnotetext{
* Corresponding author: Dr Young J Juhn, Community Pediatric \& Adolescent Medicine, Mayo Clinic, 200 First Street SW, Rochester, MN 55944,
} USA. Tel: +507-284-5243 Fax: +507-284-9744 E-mail: juhn.young@mayo.edu 
colonisation among children, because children are the major reservoir of S. pyogenes and are the target population for acute upper respiratory tract infections and complications with a peak incidence between 5 and 15 years of age. ${ }^{7}$

Known risk factors for $\mathrm{S}$. pyogenes upper respiratory tract infections include exposure to cigarette smoke, low birth weight, and low socioeconomic status. ${ }^{5,8,9}$ Recently, we found that children with asthma have an increased risk of $\mathrm{S}$. pyogenes infection compared with those without asthma. ${ }^{10}$ At present it is unknown whether atopic dermatitis or allergic rhinitis poses an increased risk of $S$. pyogenes upper respiratory tract infections. We hypothesised that children with atopic dermatitis or allergic rhinitis are more likely to develop S. pyogenes infection in the upper respiratory tract than those without such conditions due to the shared underlying immunological mechanisms between asthma and other atopic conditions.

To test this hypothesis, we conducted a retrospective cohort study to compare the incidence of S. pyogenes upper respiratory tract infections between children with and without atopic dermatitis or allergic rhinitis during the first 18 years of life. The results might help us identify the mechanisms by which some children develop S. pyogenes-induced pharyngitis and others do not, and to explain why some children become $S$. pyogenes carriers and others do not. The study findings might also enable us to understand whether the epidemiology of atopic diseases affects the epidemiology of $\mathrm{S}$. pyogenes infection. Addressing this question is worthwhile, given the significant proportion of affected children and adults with atopic dermatitis or allergic rhinitis and the rising prevalence of these conditions (10-19\% prevalence or 31.6 million Americans with atopic dermatitis and $26-33 \%$ prevalence or 60 million Americans with allergic rhinitis). ${ }^{11,12}$

\section{Methods}

\section{Study design and setting}

We conducted a retrospective cohort study of children aged 5-13 years residing in Olmsted County, Minnesota where medical care is virtually self-contained within two medical centres. They are joined by the database of the Rochester Epidemiology Project for research. A recent study on the prevalence of asthma among children and adolescents in kindergarten to 12th grades in Olmsted County, Minnesota showed that $18 \%$ of children and adolescents in this age group had asthma based on predetermined criteria upon medical record review. ${ }^{13}$

\section{Study subjects}

The study protocol was approved by the institutional review boards at both Mayo Clinic and Olmsted Medical Center. We have previously reported the details of the study subjects. ${ }^{14}$ Briefly, they were a random sample of the original study cohort of children who participated in the Rochester Family Measles Project. The original study cohort was a convenience sample of 876 children aged 5-13 years who were recruited from the
Rochester School District in Rochester, Minnesota in $1993 .{ }^{10}$ Of the original study cohort, a stratified random sample of 340 children had undergone human leucocyte antigen typing. ${ }^{15}$ These 340 children were enrolled in our current study between 2002 and 2006 when they were aged $\geq 14$ years. Subjects who did not grant research authorisation for medical record review and those who were not Olmsted County residents for at least 1 year before study enrolment were excluded. Overall, our study subjects were similar to the original study cohort and children in Olmsted County, Minnesota with regard to sociodemographic characteristics.

\section{Identification of S.pyogenes}

Details of the method for identifying $S$. pyogenes have been described previously. ${ }^{10}$ Although we did not include clinical symptoms or signs for upper respiratory tract infections as the definition of $S$. pyogenes infection, according to our medical record review almost all subjects had clinical symptoms for upper respiratory tract infections. Infection with $S$. pyogenes was ascertained by laboratory testing of a patient's throat swab. Each subject's medical records from birth to their 18th birthday were reviewed. All incidents of $S$. pyogenes culture, rapid antigen detection test, or polymerase chain reaction (PCR) test for a throat swab were recorded by date together with the result (positive or negative). We made no distinction between positive test results obtained from a throat culture, rapid antigen detection test, or PCR in data analysis. All negative results were based on either bacterial culture or PCR detection test for $S$. pyogenes.

\section{Atopic disease status}

Comprehensive medical record reviews were conducted for the study subjects to determine atopic conditions, which included atopic dermatitis or eczema and allergic rhinitis or hay fever. Ascertainment of these atopic conditions was based on the physician diagnosis of 'atopic dermatitis or eczema' and 'allergic rhinitis or hay fever' after conducting the comprehensive medical record review. We have previously used this ascertainment method for atopic conditions. ${ }^{16}$

\section{Statistical analysis}

To address the study aim, we calculated the incidence density rates by dividing the number of $S$. pyogenes upper respiratory tract infections by the total person-years based on the follow-up duration from the first registration date to the last follow-up date or 18th birthday, whichever came first. All positive test results for S. pyogenes were considered incidents of $\mathrm{S}$. pyogenes infections. The denominator (total person-years) of the incidence density rate offsets the different number of $S$. pyogenes infections, which is a function of the duration of follow-up. The incidence density rates follow the Poisson distribution since the responses were counted, so we fitted data to a Poisson regression model to calculate risk ratios and their corresponding 95\% confidence intervals, adjusting for pertinent covariates and confounders. Univariate Poisson regression was used to identify variables associated with an increased risk of $S$. pyogenes infections. Data 
were fitted to a multivariate Poisson regression model to determine the independent impact of atopic conditions on the risk of $S$. pyogenes infections, controlling for pertinent covariates and confounders (asthma status, birth weight, and ethnicity) associated with the risk of $S$. pyogenes infections. The choice of variables to be entered into a multivariate model was based on the univariate Poisson regression models and the Greenland's entering criteria for a multivariate model. ${ }^{17}$ In addition, to assess potential detection bias (i.e. atopic patients might be more likely to seek medical evaluations and tests for $S$. pyogenes infections than non-atopic patients), we compared the incidence of $S$. pyogenes infections before and after the physician diagnosis of atopic conditions. We also compared the incidence of tests for $S$. pyogenes infections, including both positive and negative test results, before and after a physician diagnosis of atopic conditions. All statistical significance was tested at a two-sided alpha error of 0.05 .

\section{Results}

\section{Study subjects}

Of the 340 children who met the eligibility criteria, only 327 were included due to lack of research authorisation for 13 children. The overall incidence of $S$. pyogenes upper respiratory tract infections in our study subjects was 0.21 per person-year. The subjects included 168 (51.4\%) males and 306 (93.6\%) Caucasians, which is similar to the overall demographics of Olmsted County in 1990. Of the 327 subjects, 143 (44\%) had atopic dermatitis or allergic rhinitis. Of these 143 subjects, 63 had atopic dermatitis (presence of asthma 28; absence of asthma 35), 110 subjects had allergic rhinitis (presence of asthma 60; absence of asthma 50), and 30 subjects had both conditions (presence of asthma 20; absence of asthma 10). In addition, of these 327 subjects, 114 met the criteria for asthma. The characteristics of the study subjects are summarised in Table 1.

Table 1. Characteristics of study subjects and factors associated with Streptococcus pyogenes infections based on a univariate Poisson regression model

\begin{tabular}{|c|c|c|c|}
\hline Characteristics & No. (\%) & $\begin{array}{l}\text { S. pyogenes incidence rate } \\
\text { (per person-year) }(95 \% \mathrm{Cl})\end{array}$ & $\begin{array}{l}\text { Unadjusted risk ratios } \\
(95 \% \mathrm{Cl})\end{array}$ \\
\hline \multicolumn{4}{|l|}{ Asthma status* } \\
\hline No & $213(65 \%)$ & $0.18(0.17$ to 0.20$)$ & Reference group \\
\hline Yes & $114(35 \%)$ & $0.25(0.23$ to 0.28$)$ & $1.38(1.12$ to 1.70$)$ \\
\hline \multicolumn{4}{|c|}{ Atopic conditions other than asthma } \\
\hline No & $184(56 \%)$ & $0.18(0.17$ to 0.20$)$ & Reference group \\
\hline Yes & $143(44 \%)$ & $0.24(0.23$ to 0.26$)$ & $1.36(1.10$ to 1.67$)$ \\
\hline \multicolumn{4}{|l|}{ Sex } \\
\hline Female & $159(49 \%)$ & $0.21(0.19$ to 0.22$)$ & Reference group \\
\hline Male & $168(51 \%)$ & $0.21(0.20$ to 0.23$)$ & $0.97(0.78$ to 1.19$)$ \\
\hline \multicolumn{4}{|l|}{ Race } \\
\hline Non-Caucasian & $21(6 \%)$ & $0.08(0.05$ to 0.11$)$ & Reference group \\
\hline Caucasian & $306(94 \%)$ & $0.22(0.20$ to 0.23$)$ & $2.89(1.41$ to 5.95$)$ \\
\hline \multicolumn{4}{|l|}{ Maternal education } \\
\hline Unknown & $110(34 \%)$ & $0.17(0.14$ to 0.21$)$ & Reference group \\
\hline High school or less & $23(7 \%)$ & $0.21(0.19$ to 0.23$)$ & $1.22(0.83$ to 1.79$)$ \\
\hline Some college & $108(33 \%)$ & $0.21(0.18$ to 0.25$)$ & $1.23(0.78$ to 1.94$)$ \\
\hline College degree & $49(15 \%)$ & $0.25(0.21$ to 0.29$)$ & $1.43(0.90$ to 2.27$)$ \\
\hline Graduate degree & $37(11 \%)$ & & \\
\hline \multicolumn{4}{|l|}{ Family history of asthma } \\
\hline No & $129(39 \%)$ & 0.20 (0.18 to 0.22$)$ & Reference group \\
\hline Yes & $198(61 \%)$ & $0.22(0.20$ to 0.23$)$ & $1.10(0.88$ to 1.36$)$ \\
\hline \multicolumn{4}{|l|}{ Family history of atopy } \\
\hline No & $106(32 \%)$ & 0.20 (0.18 to 0.22$)$ & Reference group \\
\hline Yes & $221(68 \%)$ & $0.21(0.20$ to 0.23$)$ & $1.06(0.85$ to 1.33$)$ \\
\hline \multicolumn{4}{|l|}{ Birth weight } \\
\hline Unknown & $2(1 \%)$ & $0.22(0.21$ to 0.24$)$ & Reference group \\
\hline$>2500 \mathrm{~g}$ & $255(78 \%)$ & $0.15(0.13$ to 0.17$)$ & $0.66(0.49$ to 1.89$)$ \\
\hline$<2500 \mathrm{~g}$ & $70(21 \%)$ & & \\
\hline \multicolumn{4}{|l|}{ Severity of asthma } \\
\hline Unknown & $10(8.8 \%)$ & & \\
\hline Mild to moderate & $94(82.5 \%)$ & $0.25(0.23$ to 0.28$)$ & Reference group \\
\hline Severe & $10(8.8 \%)$ & $0.28(0.21$ to 0.36$)$ & 1.12 (0.63 to 1.97$)$ \\
\hline
\end{tabular}




\begin{tabular}{|c|c|c|}
\hline & $\begin{array}{l}\text { Incidence of } \\
\text { S. pyogenes } \\
\text { infections per } \\
\text { person-year }\end{array}$ & $\begin{array}{l}\text { Adjusted risk } \\
\text { ratio }(95 \% \mathrm{Cl})^{*}\end{array}$ \\
\hline \multicolumn{3}{|c|}{ All atopic conditions } \\
\hline Yes $(n=143)$ & $0.24(0.23$ to 0.26$)$ & $1.30(1.05$ to 1.62$)$ \\
\hline No $(n=184)$ & $0.18(0.17$ to 0.20$)$ & Referent \\
\hline \multicolumn{3}{|c|}{ Atopic dermatitis or eczema } \\
\hline Yes $(n=93)$ & $0.24(0.20$ to 0.27$)$ & $1.30(0.98$ to 1.71$)$ \\
\hline No $(n=234)$ & $0.17(0.16$ to 0.21$)$ & Referent \\
\hline \multicolumn{3}{|c|}{ Allergic rhinitis or hay fever } \\
\hline Yes $(n=110)$ & $0.26(0.24$ to 0.28$)$ & $1.36(1.07$ to 1.66$)$ \\
\hline No $(n=217)$ & $0.18(0.17$ to 0.20$)$ & Referent \\
\hline
\end{tabular}

Atopic dermatitis/allergic rhinitis and S. pyogenes upper respiratory tract infection

The incidence rates of $S$. pyogenes infection for children with and without atopic dermatitis or allergic rhinitis were 0.24 per personyear and 0.18 per person-year, respectively. A multivariate Poisson regression model showed the adjusted risk ratio of patients with atopic dermatitis/allergic rhinitis developing $S$. pyogenes infection was $1.30(95 \% \mathrm{Cl} 1.05$ to $1.62, \mathrm{p}=0.018)$ compared with those without such conditions, controlling for asthma status, birth weight, and ethnicity (Table 2). We performed separate analyses for individual atopic conditions such as atopic dermatitis and allergic rhinitis. Allergic rhinitis and atopic dermatitis were found to be individually associated with increased risks of $S$. pyogenes infections adjusting for the same variables, but atopic dermatitis did not reach statistical significance (Table 2). The univariate analysis results for other factors associated with S. pyogenes infections are summarised in Table 1. To ensure that this association was not due to detection bias - that is, a differential detection of outcomes (S. pyogenes infections) between comparison groups (atopic condition status) by subjects or examiners - we compared the incidences of both positive $S$. pyogenes infections and tests for $S$. pyogenes infections regardless of the test results for subjects with atopic conditions before and after the physician diagnosis of atopic conditions. We did not find significant differences in the incidence of positive $S$. pyogenes infections ( 0.28 vs. 0.22 per person-year, respectively, $p=0.81$ ) and tests for $S$. pyogenes infections ( 0.87 vs. 0.83 per person-year, respectively, $p=0.61$ ) before and after the date of physician diagnosis of atopic conditions.

\section{Disaussion}

\section{Main findings}

Our study results suggest that children with allergic rhinitis have an increased risk of $S$. pyogenes upper respiratory tract infections compared with those without such conditions (adjusted RR $1.3,95 \% \mathrm{Cl} 1.05$ to $1.62, \mathrm{p}=0.018$ ) taking into account asthma status, ethnicity, and birth weight. Individually, allergic rhinitis (adjusted RR 1.36, 95\% Cl 1.07 to 1.66, $p=0.011$ ) was associated with an increased risk of $S$. pyogenes infections controlling for the same variables, but atopic dermatitis did not reach statistical significance due to a small sample size (adjusted RR $1.3,95 \% \mathrm{Cl} 0.98$ to 1.71 , $p=0.06$ ). Although the effect size is relatively small, we believe that the effect sizes are still significant considering the reported effect size of the known risk factor for respiratory health such as exposure to cigarette smoking (OR1.24-1.72) $)^{18,19}$ in young children. Our findings could be due to a detection bias that arises from a differential medical evaluation or medical care-seeking behaviour between children with and without atopic conditions. However, neither the incidence of $S$. pyogenes infection nor the incidence of tests for $S$. pyogenes before and after the physician diagnosis of atopic conditions is different in our study cohort. In addition, our recent study results based on a prospective cohort study showed that parents of children with asthma were not more likely to seek medical evaluations for mild acute illnesses in their children than parents of children who do not have asthma. ${ }^{20}$ These results are likely to be applicable to other atopic conditions. Our finding that the risk of $S$. pyogenes infection is independent of the timing of the physician diagnosis of atopic conditions may also suggest that the immunogenetic predisposition to atopic conditions itself may place an individual at an increased risk of $S$. pyogenes infection before developing full-blown phenotypic (clinical) characteristics of atopic conditions. Alternatively, our findings could also be due to the influence of asthma status since asthma is a common co-morbid condition, given the reported association between asthma and $S$. pyogenes infections. However, we adjusted the association between atopic dermatitis or allergic rhinitis and S. pyogenes infections for asthma status. Although we did not collect data on the use of topical corticosteroid treatment for atopic dermatitis or corticosteroid spray for allergic rhinitis, we assessed the influence of an effect of inhaled or short-term systemic corticosteroids on S. pyogenes infection among children with asthma. Only $15(13 \%)$ of the children with asthma in our study ever used inhaled or short-term systemic corticosteroids and none of them developed S. pyogenes infections in the 30 days following steroid use. A previous study showed that corticosteroid nasal spray did not increase the risk of bacterial colonisation or infection. ${ }^{21}$

Interpretation of findings in relation to previously published work

There is no literature with which we can compare our study results. We did find a single cross-sectional study that assessed the association between asthma or allergic rhinitis and S. pyogenes carrier status; this study reported an odds ratio of $1.36(95 \% \mathrm{Cl} 0.72$ to 2.57$)$ for the association. ${ }^{22}$ This 
odds ratio is similar to ours, but our sample size gives a narrower confidence interval. Given our study design as a cohort study, our study results may be more suitable to assess the association. In support of our study findings, a recent study showed a significantly increased risk of serious pneumococcal disease among children and adults with atopic dermatitis or allergic rhinitis. ${ }^{23} \mathrm{~A}$ recent study reported the prevalence of streptococcal pharyngitis and carriage in children as $12-37 \%,{ }^{24}$ but the results of this study are difficult to compare with ours (18-24\% per person-year) since we assessed the incidence of $S$. pyogenes clinical infection instead of the prevalence of infection or carriage and we used a community-based cohort rather than convenience sampling at clinics.

The mechanisms underlying the increased risk of $S$. pyogenes upper respiratory tract infections in children with allergic rhinitis are unknown. The primary mechanism might be related to increased susceptibility to bacterial colonisation and infection in patients with allergic rhinitis. ${ }^{25}$ Recent studies suggest that the filaggrin gene (heritable epithelial barrier) defect is associated with atopic dermatitis and potentially accounts for diminished epidermal defence mechanisms against microbial organisms and allergens. ${ }^{26}$ The association between filaggrin gene mutation and the increased risk of allergic rhinitis has been also demonstrated. ${ }^{27} \mathrm{~A}$ potential involvement of filaggrin in atopic marching (progression of atopic disease from atopic dermatitis to asthma and/or allergic rhinitis) has been suggested based on the predictive role of filaggrin gene mutation in the development of asthma. ${ }^{26}$ Importantly, Th2 cytokines such as interleukin (IL)-4 and IL-13 significantly downregulate filaggrin gene expression which makes the skin more susceptible to infection and allergic sensitization. ${ }^{28}$ Alternatively, Th2 cytokine gene polymorphisms might be associated with humoral immune functions such as pneumococcal antibody responses. ${ }^{29}$ Patients with allergic rhinitis might therefore have suboptimal innate and adapted immunity and be more susceptible to (impaired clearance of) microbial infections including $S$. pyogenes than those without atopic conditions.

Implications for future research, policy and practice As a potential clinical implication, given the higher risk of $S$. pyogenes infection among children with allergic rhinitis, its potential adverse outcomes, common co-infection with $\mathrm{S}$. aureus, and their potential roles in exacerbation of atopic conditions, children with poorly controlled atopic conditions including asthma may need to be screened for infections or colonisation with S. pyogenes or Staphylococcus aureus for proper treatment. ${ }^{30} \mathrm{~A}$ long-term study that assesses the effects of eradication of these bacterial organisms in airways on the control status of atopic conditions may be worthw hile. Strengths and limitations of this study

The strengths of our study include the self-contained healthcare environment and unified medical records for research among healthcare providers in our study setting. Our study assessed the long-term risk and the incidence of $S$. pyogenes infections in children with atopic dermatitis or allergic rhinitis during their first 18 years of life. Our study has an inherent limitation as a retrospective observational study, suggesting an association not a causal relationship. Also, all pertinent risk factors such as exposure to cigarette smoking in the household and allergic sensitisation status were not available. Another limitation of our study is our inability to differentiate between $S$. pyogenes carriage and infection. However, almost all instances of testing for S. pyogenes in our study subjects were due to clinical symptoms or signs. Thus, positive $S$. pyogenes tests reasonably represent clinical infections. How ever, we do not know how many patients with S. pyogenes colonisation developed clinical infections and whether children with atopic conditions are susceptible to acquiring new $S$. pyogenes infection or onset of clinical infections from colonisation is not known. Our study subjects represented predominantly a Caucasian population. Although this should not affect the interpretation of our results, it may limit generalisability to other study settings. However, the incidence of $S$. pyogenes infection in children in our study (0.21 per person-year) was similar to that reported by others (0.25 per person-year). ${ }^{9}$

\section{Conclusions}

In addition to asthma, allergic rhinitis is also associated with an increased risk of $S$. pyogenes upper respiratory tract infections. The risk of $S$. pyogenes infection is independent of asthma status and the timing of the physician diagnosis of the atopic conditions. These results suggest that the immunogenetic predisposition to atopic conditions may underlie the increased risk of $S$. pyogenes infection. Further studies are needed to elucidate the mechanisms underlying the increased risk of $S$. pyogenes upper respiratory tract infections.

\section{Handling editor Osman Mohammed Yusuf Statistical review Gopal Netuveli}

Acknowledgements We are grateful for advice and comments from Dr Gregory Poland and administrative support from Elizabeth Krusemark. We are also grateful for research support from the staff of the Pediatric Asthma Epidemiology Research Unit.

Conflicts of interest The authors declare that they have no conflicts of interest in relation to this article.

Contributorship All authors are responsible for the reported research and have participated in the concept and design, analysis and interpretation of data, drafting the manuscript, and have approved the manuscript.

Funding The research work was funded by the Scholarly Clinician Award from Mayo Clinic and made possible by the Rochester Epidemiology Project (R01-AR30582) from the National Institute of Arthritis and Musculoskeletal and Skin Diseases.

\section{References}

1. Carapetis JR, Steer AC, Mulholland EK, Weber M. The global burden of group A streptococcal diseases. Lancet Infect Dis 2005;5(11):685-94. http://dx.doi.org/ 10.1016/S1473-3099(05)70267-X

2. Linder JA, Bates DW, Lee GM, Finkelstein JA. Antibiotic treatment of children with sore throat.[see comment]. JAM A 2005;294(18):2315-22. http://dx.doi.org/ 10.1001/jama.294.18.2315

3. The World Health Organization. The World Health Organization Report 2004: 
Changing history. http://www.who.int/whr/2004/annex/topic/en/annex_2_en.pdf: WHO; 2004: http://whqlibdoc.who.int/whr/2004/924156265X.pdf.

4. The Center for Disease Control and Prevention. Bacterial Coinfections in Lung Tissue Specimens From Fatal Cases of 2009 Pandemic Influenza A (H1N1)--United States, May-August 2009. JAM A 2009;302(17):1852-4.

5. Steer AC, Danchin MH, Carapetis JR. Group A streptococcal infections in children. J Paediatr Child Health 2007;43(4):203-13. http://dx.doi.org/10.1111/j.1440-1754.2007.01051.x

6. O'Loughlin RE, Roberson A, Cieslak Paul R, et al. The epidemiology of invasive group a streptococcal infection and potential vaccine implications: United States, 20002004. Clin Infect Dis 2007;45(7):853-62. http://dx.doi.org/10.1086/521264

7. Hviid A, Melbye M. The impact of birth weight on infectious disease hospitalization in childhood. Am J Epidemiol 2007;165(7):756-61. http://dx.doi.org/10.1093/aje/kwk064

8. Nandi S, Kumar R, Ray P, Vohra H, Ganguly NK. Group A streptococcal sore throat in a periurban population of northern India: a one-year prospective study. Bull World Health Organ 2001;79(6):528-33.

9. Danchin $\mathrm{MH}$, Rogers $\mathrm{S}$, Kelpie $\mathrm{L}$, et al. Burden of acute sore throat and group a streptococcal pharyngitis in school-aged children and their families in Australia. Pediatrics 2007;120(5):950-7. http://dx.doi.org/10.1542/peds.2006-3368

10. Frey $D$, Jacobson R, Poland G, Li X, Juhn Y. Assessment of the association between pediatric asthma and $\mathrm{S}$. pyogenes upper respiratory infection. Allergy Asthma Proc 2009;30.540-5. http://dx.doi.org/10.2500/aap.2009.30.3268

11. Hanifin JM, Reed ML, Eczema Prevalence and Impact Working Group. A populationbased survey of eczema prevalence in the United States. Dermatitis 2007;18(2):8291. http://dx.doi.org/10.2310/6620.2007.06034

12. Eli OM, Michael SB, Derebery MJ, et al. Burden of allergic rhinitis: results from the Pediatric Allergies in America survey. J Allergy Clin Immunol 2009;124(3):S43-70. http://dx.doi.org/10.1016/j.jaci.2009.05.013

13. Yawn BP, Wollan P, Kurland M, Scanlon P. A longitudinal study of the prevalence of asthma in a community population of school-age children. I Pediatr 2002;140(5):576-81. http://dx.doi.org/10.1067/mpd.2002.123764

14. Juhn YJ, Kita H, Lee LA, et al. Childhood asthma and measles vaccine response. Ann Allergy Asthma Immunol 2006;97(4):469-76. http://dx.doi.org/10.1016/S1081-1206(10)60937-4

15. Poland G, Ovsyanniikova I, Jacobson $R$, et al. Identification of an association between HLA class II alleles and low antibody after measles immunization. Vaccine 2002;20.430-8. http://dx.doi.org/10.1016/S0264-410X(01)00346-2

16. Jung JA, Kita H, Yawn BP, et al. Increased risk of serious pneumococcal disease in patients with atopic conditions other than asthma. J Allergy Clin Immunol
2010;125(1):217-21. http://dx.doi.org/10.1016/j.jaci.2009.10.045

17. Greenland S. Modeling and variable selection in epidemiologic analysis. Am J Public Health 1989;79(3):340-9. http://dx.doi.org/10.2105/AJPH.79.3.340

18. Strachan DP, Cook DG. Health effects of passive smoking. 1. Parental smoking and lower respiratory illness in infancy and early childhood. Thorax 1997;52(10):905-14. http://dx.doi.org/10.1136/thx.52.10.905

19. Institute of Medicine. Clearing the Air: Asthma and Indoor Air Exposures. New York: National Academy of Sciences; 2000.

20. Juhn YJ, Johnson SK, Hashikawa AH, et al. The potential biases in studying the relationship between asthma and microbial infection. J Asthma 2007;44(10):827-32. http://dx.doi.org/10.1080/02770900701743804

21. Yilmaz F, Karabay O, Talay F, Koybasi S, Kocoglu E. The effect of triamcinolone acetonide aqueous nasal spray on the nasal carriage of Staphylococcus aureus. Am J Rhinol 2006;20(3):248-50. http://dx.doi.org/10.2500/ajr.2006.20.2866

22. Del Carmen Trojavchich M, Crisci CD, Shafa M, Rybicki BA. Relationship between group A beta-hemolytic streptococcal tonsillopharyngitis and asthma. Pediatr Allergy Immunol 2003;14(1):50-4. http://dx.doi.org/10.1034/j.1399-3038.2003.02070.x

23. Jung J, Kita H, Yawn B, et al. Increased risk of serious pneumococcal disease in patients with atopic conditions other than asthma. J Allergy Clin Immunol 2009;125:217-21.

24. Shaikh N, Leonard E, Martin JM. Prevalence of streptococcal pharyngitis and streptococcal carriage in children: a meta-analysis. Pediatrics 2010;126(3):e557-64. http://dx.doi.org/10.1542/peds.2009-2648

25. Okano M, Takishita $T$, Yamamoto $T$, et al. Presence and characterization of sensitization to staphylococcal enterotoxins in patients with allergic rhinitis. Am J Rhinol 2001;15(6):417-21.

26. Howell MD, Kim BE, Gao P, et al. Cytokine modulation of atopic dermatitis filaggrin skin expression. J Allergy Clin Immunol 2007;120(1):150-5. http://dx.doi.org/ 10.1016/j.jaci.2007.04.031

27. van den Oord RAHM, Sheikh A. Filaggrin gene defects and risk of developing allergic sensitisation and allergic disorders: systematic review and meta-analysis (see comment). BMJ 2009;339:b2433. http://dx.doi.org/10.1136/bmj.b2433

28. Ingo M, Tamara K, Anja B, et al. An interaction between filaggrin mutations and early food sensitization improves the prediction of childhood asthma. J Allergy Clin Immunol 2009;123(4):911-16. http://dx.doi.org/10.1016/j.jaci.2009.01.051

29. Wiertsema SP, Baynam G, Khoo S-K, et al. Impact of genetic variants in IL-4, IL-4RA and IL-13 on the anti-pneumococcal antibody response. Vaccine 2007;25(2):306-13. http://dx.doi.org/10.1016/j.vaccine.2006.07.024

30. David T], Cambridge GC. Bacterial infection and atopic eczema. Arch Dis Child 1986;61(1):20-3. http://dx.doi.org/10.1136/adc.61.1.20 